\title{
Epithelial Cell Transforming 2 and Aurora Kinase B Modulate Formation of Stress Granule-Containing Transcripts from Diverse Cellular Pathways in Astrocytoma Cells
}

\author{
Adrienne Weeks, ${ }^{\dagger}$ Sameer Agnihotri, ${ }^{*}$ Jennifer Lymer, ${ }^{*}$ Alan Chalil, ${ }^{*}$ Roberto Diaz, ${ }^{*}$ Semra Isik, ${ }^{*}$ Christian Smith, ${ }^{*}$ and \\ James T. Rutka*
}

From the Division of Neurosurgery, * Arthur and Sonia Labatt Brain Tumour Research Centre, Hospital for Sick Children, Toronto, Ontario; the Department of Surgery, ${ }^{\ddagger}$ University of Toronto, Toronto, Ontario; and the Division of Neurosurgery, ${ }^{\dagger}$ Department of Surgery, Dalhousie University, Halifax, Nova Scotia, Canada

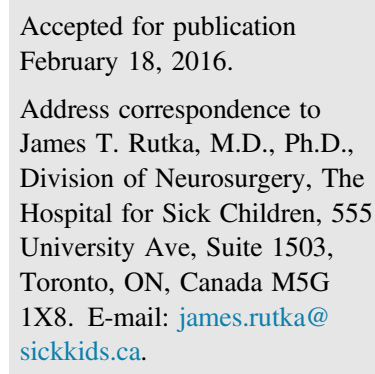

\begin{abstract}
Stress granules are small RNA-protein granules that modify the translational landscape during cellular stress to promote survival. The RhoGTPase RhoA is implicated in the formation of RNA stress granules. Our data demonstrate that the cytokinetic proteins epithelial cell transforming 2 and Aurora kinase B (AurkB) are localized to stress granules in human astrocytoma cells. AurkB and its downstream target histone-3 are phosphorylated during arsenite-induced stress. Chemical (AZD1152-HQPA) and siRNA inhibition of AurkB results in fewer and smaller stress granules when analyzed using high-throughput fluorescent-based cellomics assays. RNA immunoprecipitation with the known stress granule aggregates TIAR and G3BP1 was performed on astrocytoma cells, and subsequent analysis revealed that astrocytoma stress granules harbor unique mRNAs for various cellular pathways, including cellular migration, metabolism, translation, and transcriptional regulation. Human astrocytoma cell stress granules contain mRNAs that are known to be involved in glioma signaling and the mammalian target of rapamycin pathway. These data provide evidence that RNA stress granules are a novel form of epigenetic regulation in astrocytoma cells, which may be targetable by chemical inhibitors and enhance astrocytoma susceptibility to conventional therapy, such as radiation and chemotherapy. (Am J Pathol 2016, 186: 1674-1687; http://dx.doi.org/10.1016/j.ajpath.2016.02.013)
\end{abstract}

In response to acute environmental stress, the eukaryotic cell undergoes a complex series of steps that result in the reprioritization of cellular functions. ${ }^{1}$ During the adaptive response to stress, global translation of mRNA transcripts is repressed; however, translation of specific mRNAs occurs to aid in cell survival, such as translation of heat shock proteins and transcription factor ATF4. ${ }^{2,3}$ When translational initiation is blocked acutely by cellular stress, such as hypoxia, oxidative stress, double-stranded RNA, or radiation, the cellular response involves formation of dense aggregations of untranslated mRNA and proteins, termed stress granules. ${ }^{4,5}$ This highly conserved response to various cellular stressors requires no immediate transcriptional changes and, accordingly, is largely accomplished through translational reorganization. ${ }^{6}$
The key step in triggering stress granule formation and transcriptional silencing is phosphorylation of the eukaryotic translational initiation factor $2 \mathrm{~A}$ (eIF2 $\alpha$ ) by stressspecific kinases. ${ }^{3,7}$ Phosphorylation of eIF2 $\alpha$ results in stalled translation initiation and release of $48 \mathrm{~S}$ preinitiation complexes, such as the $18 \mathrm{~S}$ small ribosomal subunit and other initiation factors. ${ }^{8}$ The stalled $48 \mathrm{~S}$ complexes then recruit prion-like proteins with self-aggregating and RNAbinding properties, such as TIAR (alias TIAL1), TIA-1,

Supported by Canadian Institute of Health Research grant MOP 74610, b.r.a.i.n.child, Meagan's Walk (Meagan Bebenek Research Institute), and Laurie Berman and Wiley Family funds for brain tumor research.

Disclosures: None declared. 
PABP1, and G3BP1, resulting in small mRNA-protein nucleates. ${ }^{9,10}$ Protein-protein interactions with non-mRNAbinding proteins are recruited to enable complex integration and signaling. ${ }^{11}$ Small stress granule aggregates begin to coalesce and form larger mature granules in a perinuclear location, a process that relies on modifications of the cytoskeleton and recruitment of molecular motors. ${ }^{12-14}$ Compounds that interfere with this process lead to smaller stress granules that fail to reach a perinuclear location. ${ }^{12,14,15}$

The mRNA complement within stress granules has remained elusive because isolating stress granules remains a challenge. It is known that mRNAs with rich AU sequences in their $3^{\prime}$-untranslated region (UTR) are targeted to stress granules and indeed TIA-1/R and other RNA-binding proteins within stress granules, such as HuR, recognize AU-rich motifs within mRNA. ${ }^{16}$ During the lifespan of a stress granule, the mRNA found within is in flux with the cytoplasm and P-bodies (sites of mRNA decay). ${ }^{3}$ Accordingly, the fate of an individual mRNA within a stress granule can be determined by transfer to a P-body for decay, transfer to the cytoplasm for translation, or stabilization for translation on resolution of the cellular stressor. ${ }^{3}$

Formation of mature stress granules is paramount to the acute adaptive response to cellular stress. Cells with adequate stress granule formation survive, and cells with impaired stress granule assembly undergo cell death. ${ }^{17}$ Because of the critical role of stress granules in cellular health, they have been implicated in many disease processes from neural degenerative disorders, acute stroke, and, recently, cancer. ${ }^{3,18}$ Thedieck et $\mathrm{al}^{19}$ recently linked the ability to form stress granules through the recruitment of mammalian target of rapamycin (mTOR) complex to the prevention of apoptosis in cancer cells. Cancer cells exposed to radiation stabilize hypoxia-inducible factor $1 \alpha$ transcripts in stress granules to aid in resistance to radiation during the reoxygenation phase..$^{20}$ It is not surprising that interference with stress granule function presents an attractive target for cancer therapeutics.

Cytoskeletal remodeling is dependent on signaling through the RhoGTPases. Interestingly, emerging evidence suggests that RhoA signaling also regulates stress granule formation. ${ }^{21,22}$ RhoA activation leads to a dynamic reorganization of the cytoskeleton during cellular migration and cytokinesis. During cytokinesis, RhoA is modulated by two key proteins, epithelial cell transforming 2 (ECT2) and Aurora kinase B (AurkB). ${ }^{23}$ Our laboratory has previously shown that ECT2 and AurkB are involved in astrocytoma pathogenesis. ${ }^{24-26}$ Interestingly, inhibition of Aurora kinases enhances the sensitivity to radiotherapy and chemotherapy in astrocytomas and other cancers; however, the underlying molecular mechanism has not been elucidated. ${ }^{27,28}$

A hallmark of cancer is the ability to evade apoptosis. ${ }^{29}$ Given that stress granule formation is key to cell survival in hypoxia and radiation-induced stress and that RhoA plays a role in stress granule formation, we postulated that other upstream regulators of RhoA, such as ECT2 and AurkB, may also play a novel role in stress granule formation in astrocytoma cells.

\section{Materials and Methods}

\section{Cell Culture}

The permanent, well-characterized human astrocytoma cell lines, U251, U343, U118, SNB19, A341, U87, and T98, and the human embryonic kidney cell line 293T were maintained in Dulbecco's modified Eagle's medium (Thermo Fisher Scientific, Waltham, MA) with 10\% fetal bovine serum (Thermo Fisher Scientific). Glioblastoma multiforme (GBM) adherent progenitor cells were a gift from Dr. Peter Dirks (The Hospital for Sick Children, Toronto, ON, Canada) and maintained in serum-free medium on laminin-coated plates for one passage before immunohistochemistry. ${ }^{30}$ Cells were maintained at $37^{\circ} \mathrm{C}$ with $5 \% \mathrm{CO}_{2}$. Unless stated, all experiments were performed with the well-characterized U251 cell line.

\section{Stress Granule Induction}

Cells were plated the day before $60 \%$ confluence and treated with $0.5 \mathrm{mmol} / \mathrm{L}$ sodium arsenite (Sigma, St. Louis, MO) in Dulbecco's modified Eagle's medium (Thermo Fisher Scientific) and $10 \%$ fetal bovine serum (Thermo Fisher Scientific) for 30 minutes before RNA immunoprecipitation (RIP) analysis, or immunohistochemistry.

\section{AZD1152-HPQA Inhibitor}

The aurora kinase inhibitor AZD1152-HQPA (Sigma) was applied at a concentration of $10 \mathrm{nmol} / \mathrm{L}$ (specific for AurkA/ $\mathrm{B}$ at this concentration) in Dulbecco's modified Eagle's medium with $10 \%$ fetal bovine serum for 30 minutes before exposure with arsenite. ${ }^{26,31}$ The medium was then changed to include $10 \mathrm{nmol} / \mathrm{L} \mathrm{AZD1} 152$ and $0.5 \mathrm{mmol} / \mathrm{L}$ arsenite for 30 minutes.

\section{Immunohistochemistry}

Cells were fixed in $4 \%$ paraformaldehyde, as described by Smith et al. ${ }^{32}$ Cells were incubated with primary antibodies diluted in $5 \%$ goat serum at dilutions listed above for 2 hours at room temperature. Cells were then washed and incubated with Alexa-Fluor 488 anti-rabbit or anti-mouse secondary antibodies (Thermo Fisher Scientific) and/or Cy3 (Jackson ImmunoResearch Laboratories, West Grove, PA) anti-rabbit or anti-mouse secondary antibodies diluted 1:500 in 5\% goat serum. Hoechst 3342 (Thermo Fisher Scientific) diluted 1:10,000 in phosphate-buffered saline was used to visualize nuclei. Cells were mounted in Dako mounting medium (Dako, Glostrup, Denmark) and visualized with a Zeiss Axiovert 200M Spinning Disk confocal 
microscope (Carl Zeiss Canada Ltd., Toronto, Canada) equipped with a Hamamatsu Back-Thinned EM-CCD camera (Hamamatsu Corp., Hamamatsu City, Japan) and analyzed using Volocity Software version 5.5 and 6.3 (PerkinElmer, Waltham, MA). Antibody concentrations were as follows: TIAR (BD Bioscience, Franklin Lakes, NJ), 1:400; G3BP1 (Millipore, Billerica, MA), 1:400; and ECT2 (Santa Cruz Biotechnology, Dallas, TX) 1:200.

\section{Coimmunoprecipitation}

U251 cellular lysate $(1 \mathrm{mg})$ was incubated with Protein-A agarose beads and $1.5 \mathrm{mg}$ of rabbit polyclonal anti-ECT2 or rabbit IgG (Santa Cruz Biotechnology) for 4 hours at $4^{\circ} \mathrm{C}$. Samples were then subjected to SDS-PAGE and Western blot analysis.

\section{siRNA Transfection}

On Target Plus SMARTpool siRNA (Thermo Fisher Scientific) of ECT2 (5'-GCACUCACCUUGUAGUUGA- $3^{\prime}, 5^{\prime}$ CAGAGGAGAUUAAGACUAU- $3^{\prime}$, 5'-UAACAGCAAUCGCAAACGA-3', 5'-GUAAUGAGUCGUCUUUCUA-3') and AurkB (5'-CAGAAGAGCUGCACAUUUG-3', $5^{\prime}$-GCGCAGAGAGAUCGAAAUC-3', 5'-ACGCGGCACUUCACAAUUG-3', 5'-CCAAACUGCUCAGGCAUAA-3') were transfected in glioma cells using Lipofectamine RNAiMAX (Thermo Fisher Scientific) and 200 pmol of each siRNA in $10 \mathrm{~mL}$. Forty-eight hours after transfection, the cells were processed for experiments.

\section{RNA Immunoprecipitation}

Cells were counted and plated 24 hours in advance in $15-\mathrm{cm}$ plates. Cells were then treated for 30 minutes with either $0.5 \mathrm{mmol} / \mathrm{L}$ sodium arsenite or regular medium. Cells were then prepared as per Magna RIP RNA binding immunoprecipitation kit (Millipore) with $2 \times 10^{7}$ cells per RIP reaction. Each reaction was performed in triplicate. Each RIP reaction $(10 \mu \mathrm{L})$ was stored as input control; $10 \mu \mathrm{g}$ of TIAR (BD Bioscience) or G3BP1 (Millipore) antibodies was used in each RIP reaction. Immunoprecipitations were performed for 4 hours.

\section{Cellomics Assay}

Using Cellomics VTI (Thermo Fisher Scientific) and a modified SPOT Detector Bioapplication (version 2; Cellomics) algorithm, cells, nuclei, and stress granules were identified on the basis of intensity thresholds between adjacent pixels. Identification of objects within an imaged field was accepted or rejected for analysis on the basis of fluorescence intensity, Hoescht nuclear dye, or TIAR. Five hundred cells were plated per well in a 96-well plate (VWR, Radnor, PA) for each condition in 12 technical replicates.
Each Cellomics assay was performed in three biological replicates.

\section{Microarrays}

Only RNA that passed analysis on the Agilent Bioanalyzer was subsequently used for microarray analysis. The RNA integrity number is a value generated using RNA aliquots and the Agilent 2100 Bioanalyzer (Agilent Technologies, Santa Clara, CA), which is a software tool designed to help estimate the integrity of total RNA samples. The Center for Applied Genomics, SickKids (Toronto, ON, Canada), performed this analysis. An RNA integrity number score of 7 or higher on a scale of 1 to 10 indicates good quality total RNA with presence of little to no degradation products. All samples that passed an RNA integrity number score $>7$ were used for microarray analysis as per the facilities protocols. Three samples of each condition of RNA immunoprecipitated from non-stressed (TIAR, G3BP1) and arsenite-treated (TIAR and G3BP1) lysates as well as non-stressed and arsenite input RNA controls were run in triplicate on Affymetrix Human exon 1.0 ST arrays by The Center for Applied Genomics (The Hospital for Sick Children, Toronto, ON, Canada).

\section{Data Analysis}

Affymetrix CEL files from Affymetrix Human Exon 1.0 ST array were then imported into Affymetrix Expression Console (version 1.1), and gene-level analysis (CORE content) was performed. Arrays were quantile normalized (RMA sketch normalization), and probe sets were annotated according to the human genome build HG19 (GRCh37). Significant genes from three individual RIP experiments were identified as follows: Percentage of input was calculated by dividing RNA expression values from TIAR or G3BP1 pull-downs by total input expression values (in stressed or non-stressed conditions). These ratios were compared with each other to identify enrichment of genes pulled down from TIAR or G3BP1 in stressed versus nonstressed conditions: Percentage Input $=$ TIAR IP (in Stress)/Input RNA (in Stress) versus TIAR IP (Non-Stress)/ Input RNA (Non-Stress).

A similar analysis was performed for G3BP1. Genes with a minimum twofold enrichment in stressed versus non-stressed conditions with $P<0.001$ and a false discovery $<5 \%$ were determined as significantly enriched under stress conditions compared with non-stressed conditions using a Bonferronicorrected $t$-test. To eliminate the effect of arsenite on gene expression, we performed a separate analysis comparing nonstress with stress inputs in triplicate. Genes with a twofold increase or greater, and significant $\alpha(P<0.001)$ were eliminated from TIAR or G3BP1. Ingenuity pathway analysis and gene-set enrichment analysis were performed on enriched genes to identify significant biological pathways, molecular processes, and activated/inhibited networks. 
Microarray data have been deposited into the Gene Expression Omnibus database (http://www.ncbi.nlm.nih. gov/geo; accession number GSE76814).

\section{Prediction of AU-Rich Elements in Candidate Genes}

The 19 enriched genes common to both TIAR and G3BP1 RIPS with arsenite treatment were analyzed with AREsite, as per Gruber et $\mathrm{al}^{33}{ }^{33}$ using the website AREsite (http:// rna.tbi.univie.ac.at/AREsite, last accessed January 26, 2016).

\section{Correlation of G3BP1 and TIAR Expression and Survival}

Using R2 Genomics software (http://r2.amc.nl, last accessed June 21, 2015), we analyzed TIAL1 and G3BP1 expression levels in The Cancer Genome Atlas (TCGA) GBM data set ${ }^{34}$ for survival differences. TIAL1 and G3BP1 RNA expression and survival data analysis was also performed independently in additional GBM and astrocytoma samples using Repository for Molecular Brain Neoplasia Data data set $^{35,36}$ with normalized gene expression data accessed through the Repository for Molecular BRAin Neoplasia DaTA data portal (REMBRANDT; http://www.betastasis.com/gliomal rembrandt, last accessed June 22, 2015). Patients were grouped according to high and low expression levels using a median RNA expression cutoff selection. Survival differences were then quantified by log-rank tests and illustrated using Kaplan-Meier estimates. GBM subtype analysis was performed on 528 samples in concordance with the Verhaak and colleagues ${ }^{34,36}$ and TCGA GBM study. Significance was established at $P<0.05$.

\section{Results}

The Upstream Regulator of RhoA, ECT2, Is Associated with RNA Stress Granules

The upstream regulator of RhoA, ECT2, is aberrantly localized in the cytoplasm in U251 astrocytoma cells. ${ }^{24}$ During unstressed conditions, cytoplasmic ECT2 is found within the cytosol and at the leading edge of astrocytoma cells, as previously described. ${ }^{24}$ The addition of arsenite for 30 minutes results in stress granule formation, as demonstrated by TIAR aggregation. ${ }^{37}$ During arsenite-induced stress, ECT2 colocalized with aggregated TIAR (Figure 1A). The Costes Pearson coefficient obtained from stress granules defined by TIAR immunofluorescence and costained with ECT2 and TIAR was $0.91 \pm 0.3 .{ }^{38}$ Immunoprecipitation of endogenous ECT2 showed increasing association with TIAR under arsenite-induced stress (Figure 1B). Because stress granules harbor untranslated mRNA sequences, RNA immunoprecipitation was performed on arsenite-treated cellular lysates with the known stress granule ribonucleotide-binding proteins TIAR, PABPC1, an IgG negative control, and ECT2. RT-PCR for $\beta$-actin after RNA immunoprecipitation demonstrated association of $\beta$-actin mRNA with ECT2 and the positive controls, but not the negative $\operatorname{IgG}$ control (Figure 1C).

siRNA-Mediated Silencing of ECT2 Results in Changes in Stress Granule Localization and Size but Not Number of Stress Granules

Both 20 and $40 \mathrm{nmol} / \mathrm{L}$ concentrations of pooled ECT2 or nonsilencing (NS) siRNA were effectively transfected into U251 astrocytoma cells (Figure 1D). For all further experiments, $20 \mathrm{nmol} / \mathrm{L}$ of pooled siRNA was used. Highthroughput cellomics-based spot counting assays were performed using TIAR fluorescence in ECT2 siRNA or NS siRNA exposed U251 cells with and without arsenite treatment. ECT2 is essential for cytokinesis and treatment with ECT2 siRNA; as previously published, it resulted in significantly larger binucleate cells (Supplemental Figure S1, A and B) after 48 hours. ${ }^{39-41}$ To account for increased cytoplasmic volume in ECT2 siRNA cells, stress granule number was measured as stress granules per $\mu \mathrm{m}^{2}$. Stress granule size (in pixels ${ }^{2}$ ), TIAR fluorescence pixel intensity within the stress granule aggregate, and number of stress granules within a $7-\mu \mathrm{m}$ ring from the nucleus were also calculated and analyzed. Although ECT2 siRNA did not diminish stress granule number, silencing of ECT2 resulted in fewer stress granules in a mature perinuclear location and a small, but significant, change in stress granule size (Figure 1, F-I). Representative immunofluorescence staining of TIAR in NS siRNA and ECT2 siRNA U251 cells confirmed the cellomics data that stress granules in ECT2 siRNA cells occur at a more distant perinuclear location (Figure 1G). ECT2 did not affect TIAR pixel fluorescent intensity (data not shown). Consistent with ECT2 playing a role downstream of initial stress granule formation, siRNA-mediated knockdown of ECT2 did not change the amount of phosphorylated eIF $2 \alpha$ by Western blot analysis (Supplemental Figure S1C).

\section{siRNA-Mediated Silencing of ECT2 Results in Sensitization to Doxorubicin-Mediated Apoptosis}

The chemotherapeutic agent doxorubicin is a known trigger of stress granule formation. ${ }^{42}$ We hypothesized that if ECT2 plays a role in the stress granule life cycle, then siRNAmediated silencing of ECT2 would increase the apoptotic effects of doxorubicin. Indeed, siRNA-mediated silencing of ECT2 results in increased apoptosis at baseline, and increased apoptosis with the addition of doxorubicin compared with NS siRNA controls, as shown by cleaved poly (ADP-ribose) polymerase status controls in U251 cells (Figure 1E).

AurkA and AurkB Are Phosphorylated and Active during Arsenite-Induced Stress

AurkB is immediately upstream of ECT2 in the pathway regulating cytokinesis. Given the modest effects observed 
A

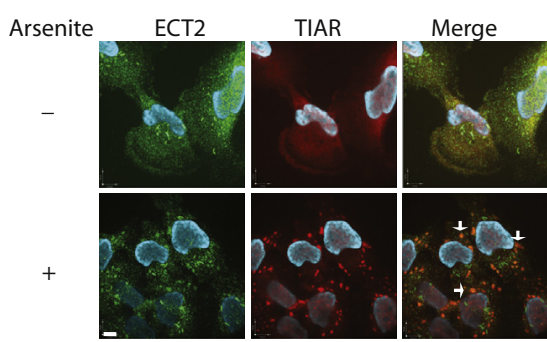

D

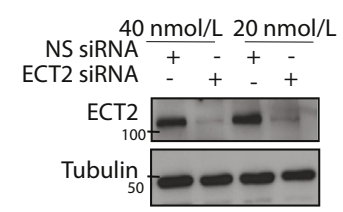

G

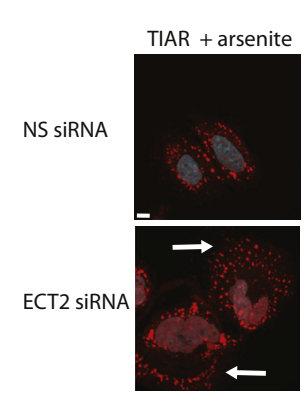

B

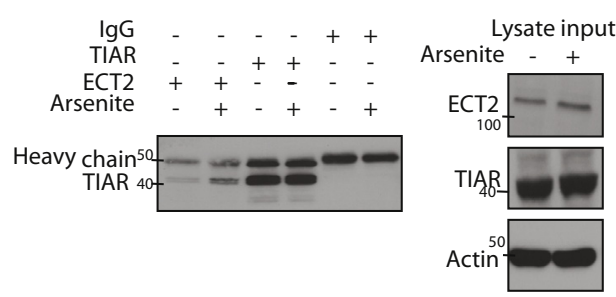

E

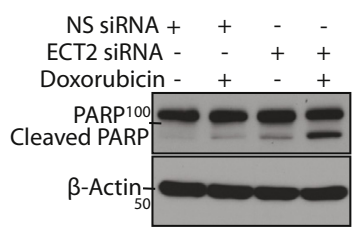

H

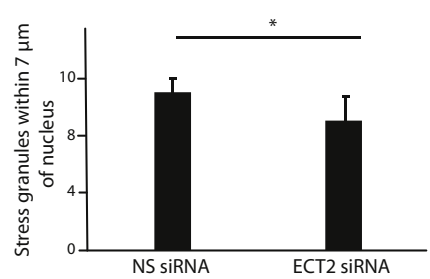

C
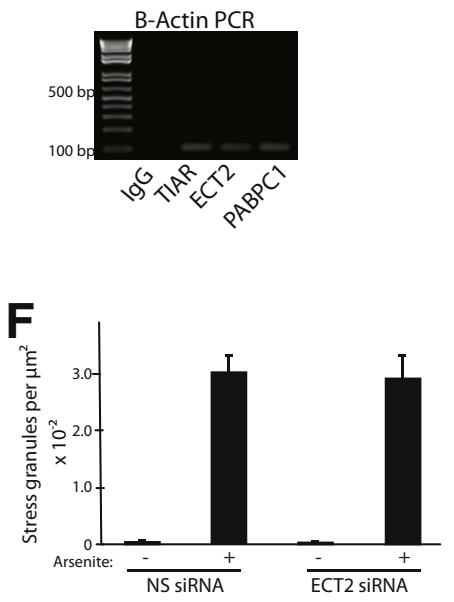

I

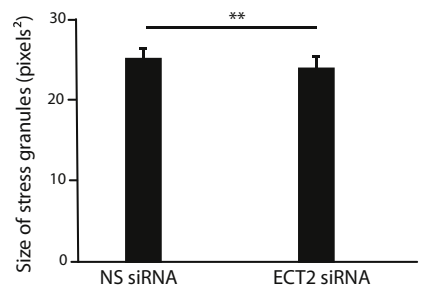

Figure 1 A: Immunofluorescence staining in U251 astrocytoma cells of epithelial cell transforming 2 (ECT2; green), TIAR (red), and merge with or without exposure to 30 minutes of $0.5 \mathrm{mmol} / \mathrm{L}$ arsenite demonstrates colocalization of ECT2 with stress-induced TIAR stress granule aggregates (arrows). B: TIAR Western blot analysis of endogenous pull-downs of ECT2, TIAR, and IgG from stressed and unstressed U251 protein lysates. The endogenous ECT2 pull-down demonstrates increasing TIAR association during arsenite-induced stress. Input lysate levels of ECT2, TIAR, and $\beta$-actin were used as loading control. C: Agarose gel showing RT-PCR for $\beta$-actin mRNA after RNA immunoprecipitation with ECT2, IgG (negative control), TIAR, and PABPC1 (positive control) in arsenite-treated U251 cells. ECT2, PABC1, and TIAR demonstrate $\beta$-actin mRNA pull-down but not the negative IgG control. D: Western blot analysis of lysates from either 20 or $40 \mathrm{nmol} / \mathrm{L}$ transfection of pooled ECT2 siRNA or nonsilencing (NS) siRNA. Both concentrations achieve efficient knockdown of ECT2 with little residual expression; $20 \mathrm{nmol} / \mathrm{L}$ of pooled ECT2 siRNA was used in all further experiments. E: Western blot demonstrating cleaved and uncleaved poly (ADPribose) polymerase (PARP) and $\beta$-actin control. Lysates from ECT2 siRNA treated cells show increased cleaved PARP at baseline and increased sensitivity to doxorubicin-induced apoptosis compared with NS siRNA controls. F: Graphical representation of high-throughput cellomics TIAR fluorescent spot assay demonstrating no significant change $(P=0.3)$ in stress granule per $\mu \mathrm{m}^{2}$ with ECT2 versus NS siRNA with and without arsenite treatment, $0.04 \pm 0.03 \times 10^{-2}$ and $0.03 \pm 0.02 \times 10^{-2}$ for NS siRNA and ECT2 siRNA without arsenite and $3.03 \pm 0.3 \times 10^{-2}$ and $2.92 \pm 0.4 \times 10^{-2}$ for NS siRNA and ECT2 siRNA with arsenite treatment, respectively. G: Immunofluorescence of TIAR stress granules after arsenite treatment in NS siRNA and ECT2 siRNA treated U251 cells demonstrating an increased peripheral localization of stress granules (arrows) in relation to the nucleus of ECT2 siRNA cells. H: Graphical representation of high-throughput cellomics TIAR fluorescent spot assay showing a statistically significant $(P=0.03)$ difference in the number of granules within $7 \mu \mathrm{m}$ of the nucleus with ECT2 siRNA versus NS siRNA, $10.8 \pm 1.1$ versus $9.1 \pm 1.8$. I: Graphical representation of high-throughput cellomics TIAR fluorescent spot assay demonstrating a small, but statistically significant $(P=0.003)$, change in the size of stress granules with ECT2 siRNA versus NS siRNA, $23.9 \pm 1.4$ and $25.1 \pm 1.3$ pixels $^{2}$ in cells treated with arsenite. ${ }^{*} P<0.05,{ }^{* *} P<0.01$. Scale bar $=7 \mu \mathrm{m}(\mathbf{A}$ and $\mathbf{G})$.

when ECT2 is silenced with siRNA, we postulated that other upstream regulators in the RhoA pathway might also contribute to the stress granule life cycle. Exposure of U251 astrocytoma cells to arsenite-induced stress resulted in phosphorylation and activation of AurkA/B. AurkA/B phosphorylation was abolished when cells were pretreated with $10 \mu \mathrm{mol} / \mathrm{L}$ of the aurora kinase inhibitor AZD1152 before exposure to arsenite (Figure 2A). Interestingly, the addition of AZD1152 also diminished the phosphorylation status of the stress granule inducer, eIF2 $\alpha$ (Figure 2A). To confirm that arsenite-induced phosphorylation of AurkB resulted in general activation of AurkB pathways, we used the phosphorylation status of a well-characterized downstream target of AurkB, phosphorylated-histone-3 (p-Histone3) as a surrogate of global AurkB activation. Levels of p-Histone3 increased over a 20-minute time course after arsenite treatment, consistent with AurkB activation (Figure 2B). AurkB colocalized with TIAR in approximately $10 \%$ of cells, as identified by immunofluorescence (Figure 2C). Using the high-throughput cellomics TIAR fluorescence spot assay, blockade of AurkA/B activation by AZD1152 30 minutes before arsenite treatment resulted in significantly fewer stress granules per cell than untreated cells exposed to arsenite (Figure 2D). 
A

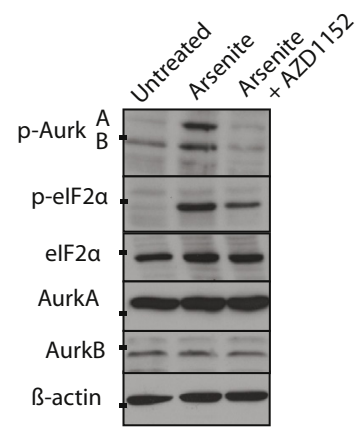

D

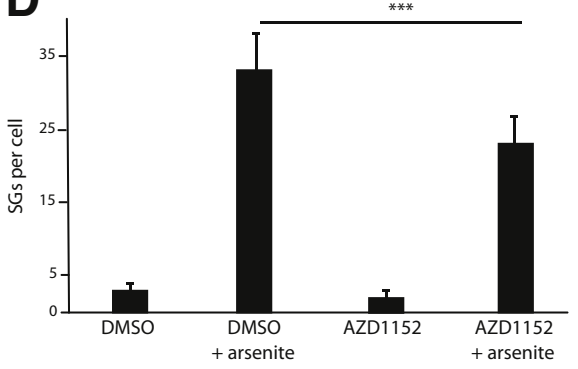

B

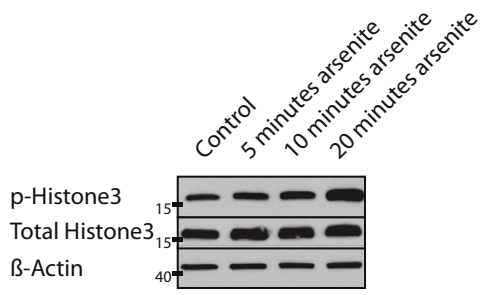

$\mathbf{E}$

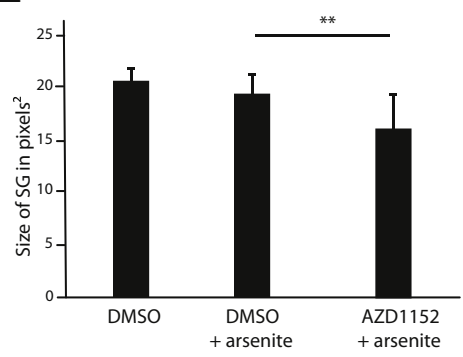

C

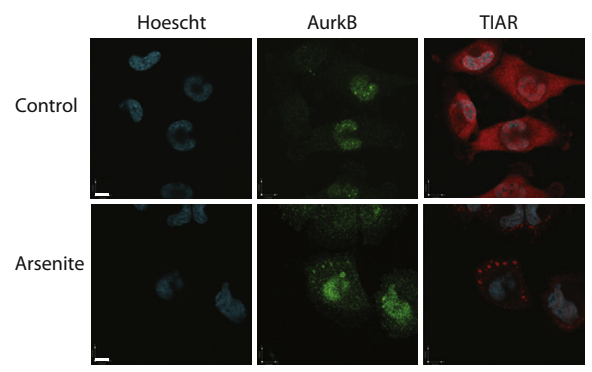

$\mathbf{F}$

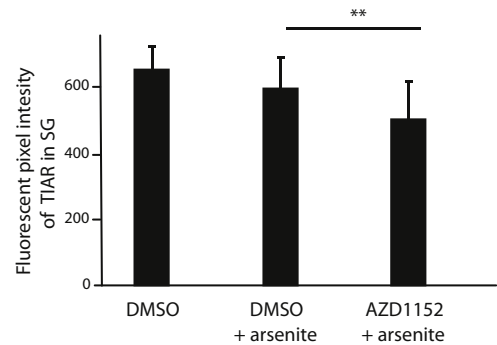

Figure 2 A: Western blot analysis demonstrating increased phosphorylation of Aurora kinase A/B (AurkA/B) and eukaryotic translational initiation factor $2 \mathrm{~A}(\mathrm{eIF} 2 \alpha)$ during stress induction with $0.5 \mathrm{mmol} / \mathrm{L}$ arsenite over 30 minutes in U251 astrocytoma cells. This phosphorylation is diminished with pretreatment of $10 \mu \mathrm{m}$ aurora kinase inhibitor AZD1152 before arsenite exposure. B: Western blot analysis of phosphorylated-histone-3 over a 20-minute arsenite stress induction time course. Total histone and $\beta$-actin are shown as loading controls. The downstream target of AurkB, histone-3, is phosphorylated over a 20 minutes time course with arsenite. C: Immunofluorescence staining of AurkB (green) and TIAR (red) with and without arsenite treatment demonstrating that AurkB and TIAR colocalize in a subpopulation of U251 cells. D: Graphical representation of high-throughput cellomics TIAR fluorescent spot assay demonstrating $32.6 \pm 4.6$ SD stress granules (SG) per cell in arsenite and dimethyl sulfoxide (DMSO) control-treated U251 cells compared with $23.7 \pm 4.2$ SD SGs per cell in U251 cells pretreated with AZD1152 before arsenite stress (two-tailed $t$-test, $P=0.0002$ ). Addition of AZD1152 does not significantly alter SGs in non-arsenite-treated cells, $3.4 \pm 1.2$ SD in DMSO alone versus $2.3 \pm 1.4$ SD in AZD1152-treated cells. E: Graphical representation of high-throughput cellomics TIAR fluorescent spot assay demonstrating a significant reduction in SG size, $16.0 \pm 3.3$ SD pixels ${ }^{2}$ in AZD1152 pretreated cells versus $21.0 \pm 1.2$ SD pixels ${ }^{2}$ (two-tailed $t$-test, $P=0.0005$ ) in arsenite controls. F: Graphical representation of high-throughput cellomics TIAR fluorescent spot assay demonstrating a significant reduction in TIAR pixel intensity in SGs with pretreatment with AZD1152 $507 \pm 125$ SD versus $659 \pm 68$ SD in controls (twotailed $t$-test, $P=0.007) .{ }^{* *} P<0.01,{ }^{* *} P<0.001$. Scale bar $=7 \mu \mathrm{m}$.

Pretreatment with AZD1152 also resulted in stress granules that were smaller and decreased TIAR fluorescence intensity (Figure 2, E and F).

\section{siRNA-Mediated Knockdown of AurkB, but Not AurkA, Reduces Stress Granule Number and Formation}

Similar to the experiments described using ECT2 siRNA, AurkB siRNA treatment over 48 hours resulted in significantly larger cells with cytokinetic failure (Supplemental Figure S1, A and B). We were able to mediate siRNA silencing of AurkB with $20 \mathrm{nmol} / \mathrm{L}$ of pooled AurkB siRNA (Figure 3A). Cellomics spot assays using TIAR fluorescence demonstrated fewer stress granules per $\mu \mathrm{m}^{2}$, diminished TIAR fluorescent intensity, and smaller stress granules in AurkB siRNA plus arsenite-treated U251 cells compared with NS siRNA plus arsenite-treated cells (Figure 3, B-D). AurkB siRNA plus arsenitetreated cells also had significantly more cells with $<5$ stress granules per cell (baseline) and significantly fewer cells with $>20$ stress granules per cell, albeit the percentage change is modest (Table 1).

\section{Astrocytoma Cell Line and Progenitor Cells Contain Stress Granules}

U251 astrocytoma cell lines express stress granules (Figure 1A). Low passage adherent GBM progenitor cells maintained in serum-free media were examined for stress granule formation to ensure stress granule formation occurs in GBM cells cultured directly from patients. Stress granules were defined as aggregates that demonstrate colocalization of TIAR and G3BP1. In addition to established cell lines (U251, U343, U118, SNB19, A341, U87, and T98, data not shown), primary GBM progenitors express stress granules after arsenite stress, as defined by colocalization of TIAR and G3BP1 immunofluorescence aggregates compared with untreated controls (Figure 4A).

\section{RNA Immunoprecipitation with TIAR and G3BP1 of Arsenite-Treated Cellular Lysates Enriches for the 18S Ribosomal Subunit}

Stress granules are not membrane-bound organelles, and attempts to isolate stress granules to better understand the 

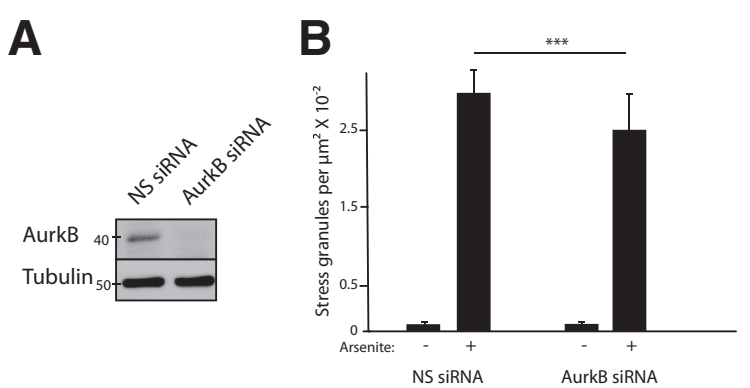
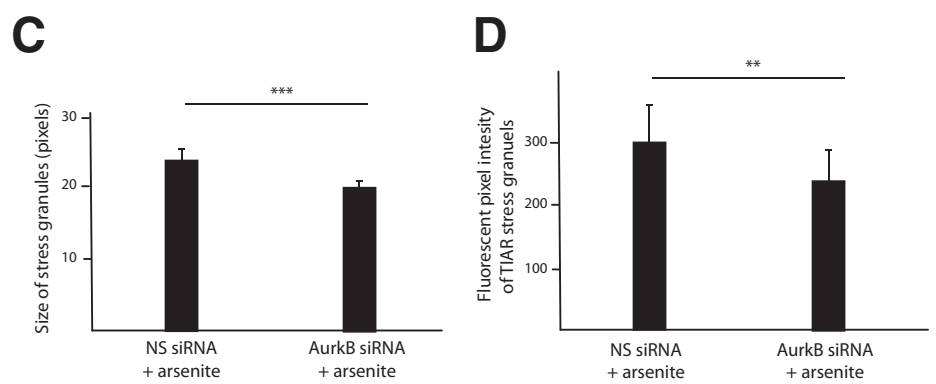

Figure 3 A: The $20 \mathrm{nmol} / \mathrm{L}$ of pooled Aurora kinase B (AurkB) siRNA significantly reduces protein expression compared with pooled nonsilencing (NS) siRNA controls. B: Graphical representation of high-throughput cellomics TIAR fluorescent spot assay demonstrating AurkB siRNA significantly reduces stress granules per $\mu \mathrm{m}^{2} \times 10^{-2}, 2.57 \pm 0.4 \mathrm{SD}$ versus NS siRNA $3.03 \pm 0.3 \mathrm{SD}$ (two-tailed $t$-test, $P=0.001$ ) in arsenite-treated U251 cells. C: Graphical representation of high-throughput cellomics TIAR fluorescent spot assay demonstrating a decrease in the average size of stress granules in AurkB siRNA arsenite-treated U251 cells (20.2 \pm 0.9 SD) versus NS siRNA arsenite-treated U251 cells (24.3 \pm 1.3 SD) (two-tailed $t$-test, $P<0.001)$. D: Graphical representation of high-throughput cellomics TIAR fluorescent spot assay demonstrating a loss of TIAR fluorescent intensity in stress granules in AurkB siRNA treated U251 cells $(238 \pm 51 \mathrm{SD}$ ) versus NS siRNA treated U251 cells (304 $\pm 56 \mathrm{SD}$ ) (two-tailed $t$-test, $P=0.01)$. ${ }^{* *} P<0.01$, $* * * P<0.001$.

protein and RNA composition have proved difficult. We performed RIPs of U251 cellular lysates in both stressed and non-stressed conditions with TIAR, G3BP1, and control IgG. Input controls in both stressed and unstressed conditions were analyzed. As expected, input lysates demonstrated both $18 \mathrm{~S}$ and 28S RNA subunits by electropherogram (Figure 4B). Electropherograms of RIPs with TIAR under stress conditions resulted in an enrichment in the $18 \mathrm{~S}$ ribosomal subunit. This result is expected because stress granules are known to contain the $18 \mathrm{~S}$ ribosomal subunit (which is a part of the small 40S initiation ribosomal subunit) but lack the $28 \mathrm{~S}$ ribosomal subunit (which is a part of the larger $60 \mathrm{~S}$ translation elongation ribosomal subunit) ${ }^{8}$ (Figure 4B). Interestingly, G3BP1 RIPs showed an increase in $18 \mathrm{~S}$ subunit in both stressed and unstressed conditions (Figure 4B). IgG RIPs showed little RNA enrichment and was not used in subsequent microarray analyses (Supplemental Figure S1E).

Microarray Analysis of RIPs with G3BP1 and TIAR under Stress and Non-Stressed Conditions Yield Candidate mRNA Contained within Stress Granules

U251 astrocytoma cells were either untreated or treated with arsenite to induce stress granule formation, and RNA

Table 1 Quantification of Stress Granules per Cell after AurkB RNAi Knockdown

\begin{tabular}{lllll}
\hline siRNA & $<5^{*}$ & $5-20^{*}$ & $>20^{*}$ & Total \\
\hline NS & 5 & 18 & 77 & 1261 \\
AurkB & 8 & 22 & $70^{\dagger}$ & 1241 \\
\hline
\end{tabular}

AurkB siRNA arsenite-treated U251 cells have a modest, but statistically significant, increase in stress granules per cell $(>20$ stress granules per cell; $P=0.003$, Fisher exact test) over baseline of $<5$ stress granules per cell.

${ }^{*}$ Data are given as percentage.

${ }^{\dagger} P<0.01$ versus NS.

AurkB, Aurora kinase B; NS, nonsilencing; RNAi, RNA interference. immunoprecipitates were analyzed by exon array analysis. Affymetrix exon array analysis of arsenite-treated astrocytoma cells revealed 108 and 769 RNA species that were enriched in TIAR RIPs and G3BP1 RIPs, respectively, compared with TIAR and G3BP1 RIPs from untreated cells and input controls (Figure 5, A and B). Ingenuity pathway analysis on the stress granule-enriched mRNAs from the TIAR and G3BP1 RIPs demonstrated significant involvement of multiple functional biology networks (Figure 5, C and D, and Supplemental Table S1). Signaling pathway analysis of the stress-induced G3BP1 and TIAR RIPs show the preponderance of elements of various signaling pathways (Figure 5, E and F), respectively. Notably, astrocytoma signaling was enriched in the stress-induced G3BP1 RIPs (Figure 5E). G3BP1 stress-induced RIPs demonstrated an enrichment of genes affected by miRNA pathways: miR-122-5p, miR-17-5p, miR-483-3p, miR-210, miR-17, and miR-124-3p (Figure 6A), whereas none of the miRNA pathways were observed in TIAR RIPs (Figure 6B). G3BP1 RIPs also had an increase in mRNA pathways affected by sirolimus (an mTOR inhibitor used in cancer therapeutics) (Figure 6B). Nineteen RNA species were common between TIAR and G3BP1 RIPs from arsenite treated cells. Of these, 8 gene products are associated with known functions in cellular stress response and include: cerebellar degeneration related protein 1 (CDR1); coxsackie virus and adenovirus receptor (CXADR); disabled 2 (DAB2); enhancer of polycomb homolog 2 (EPC2); HISTH1H3A; mitochondrial ribosomal protein L41 (MRPL41); THO complex 7 (THOC7); brain expressed X-linked 1 (BEX1). The other 11 mRNAs have yet to be linked to a function in cellular stress response and include: kinectin 1 (KTN1); limb development membrane protein 1 (LMBR1); leucine rich repeat and coiledcoil centrosomal protein 1 (LRRCC1); mitochondrial ribosomal protein L41 (MRPL41); non-coding RNA 00183 (NCRNA00183); receptor accessory protein 3 (REEP3); ribonuclease T2 (RNASET2); TGF-beta activated kinase 1/MAP3K7 binding protein 2 (TAB2); transmembrane 


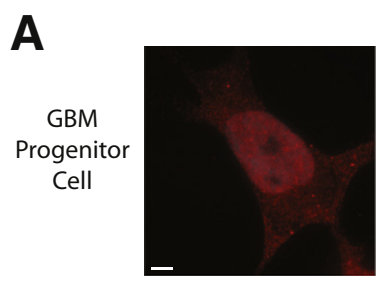

TIAR

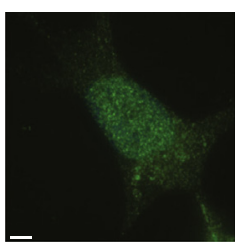

G3BP1

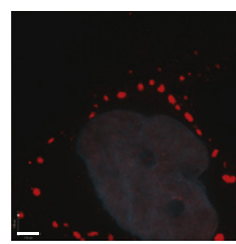

TIAR

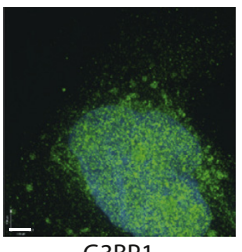

G3BP1

B

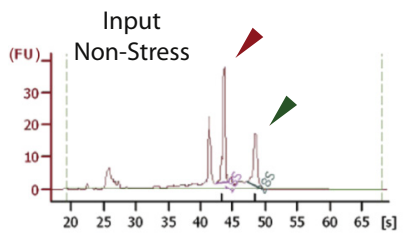

TIAR
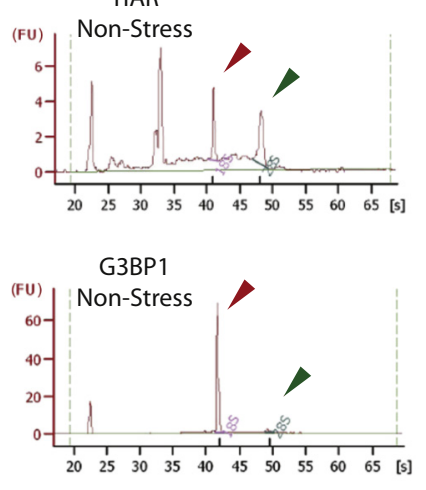
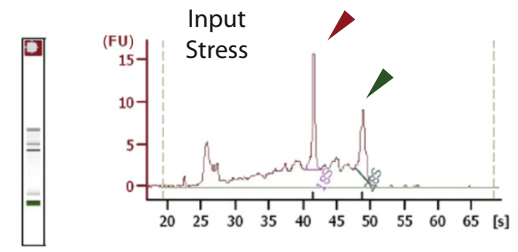

TIAR
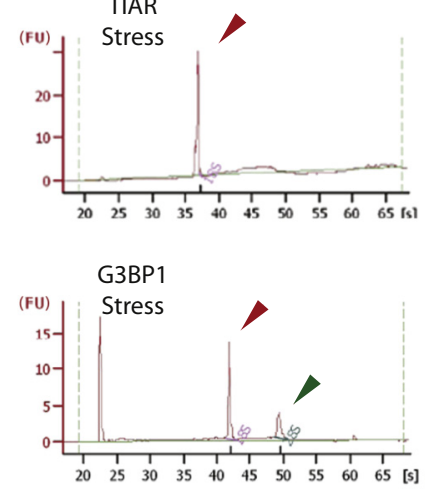

Figure 4 A: Glioblastoma multiforme (GBM) progenitor cells maintained in low passage number and serum-free media retain ability to form stress granules, as shown by TIAR and G3BP1 immunofluorescence aggregates after the addition of 0.5 $\mathrm{mmol} / \mathrm{L}$ arsenite. B: Electropherogram of RNA precipitate from cellular input lysates, TIAR RNA immunoprecipitation, and G3BP1 immunoprecipitation under both non-stressed and stressed conditions. Input lysates contained a combination of $18 \mathrm{~S}$ (red arrowheads) and 28S (green arrowheads) ribosomal subunits in both stressed and non-stressed conditions. Under arsenite-induced stress, TIAR immunoprecipitates were enriched for the 18Sribosomal subunit. G3BP1 demonstrates enrichment of the $18 \mathrm{~S}$ subunit in both stressed and unstressed conditions. Scale bars $=7 \mu \mathrm{m}$. FU, fluorescence units. protein 141 (TMEM141); translocase of outer mitochondrial membrane 20 (TOMM20); zinc finger protein 32 (ZNF32) (Figure 6C). Biological functions involved in these 19 commonly enriched genes in TIAR and G3BP1 stress RIPs include cellular movement, cellular organization, cell morphology, cell death, proliferation, RNA trafficking, and post-translational modifications (Figure 6D).

mRNA transcripts that are known to be a component of stress granules typically contain an AU-rich element in their $3^{\prime}$-UTR. ${ }^{16}$ Analysis of the 19 genes common to TIAR and G3BP1 using a comprehensive database to predict AU-rich elements in the $3^{\prime}$-UTR of mRNA demonstrated that 15 of 19 genes contained at least one of the eight AU-rich binding motifs $^{33}$ (Supplemental Table S2).

\section{G3BP1 and TIAR Expression Levels and Survival}

To evaluate the clinical aspect of G3BP1 and TIAL1 expression, we interrogated two large public glioma RNA expression data sets, REMBRANT and TCGA. In the REMBRANDT data set, high G3BP1 RNA expression was associated with a trend toward poorer overall survival in GBM and was significant in grade II to III astrocytomas $(P<0.05)$ (Figure 7, A and B). Conversely, high TIAL1 RNA expression was associated with a trend toward better overall survival in GBM and was significant in astrocytomas $(P<0.05)$ (Figure 7, C and D).
To complement the REMBRANT data set, we interrogated the significance of G3BP1 and TIAL1 RNA expression with respect to survival in the GBM TCGA data set. ${ }^{34}$ As observed in the GBM and astrocytoma REMBRANDT data set, high TIAL1 expression correlated with better overall expression in the TCGA data set compared with low TIAL1 expression $(P<0.05)$ (Supplemental Figure S2A). G3BP1 RNA expression in the TCGA data set did not significantly correlate with survival (Supplemental Figure S2B). GBM comprises several molecular subtypes, each with varying clinical and biological features. ${ }^{34,35}$ We analyzed 528 GBMs for which we had molecular subtype analysis data and observed that both G3BP1 and TIAL1 were not significantly enriched in any GBM subtype (Figure 7, E and F).

\section{Discussion}

Eukaryotic cells undergo a myriad of changes in response to acute cellular stress that determine the balance of activation of prosurvival pathways or cell death pathways, such as apoptosis, autophagy, and necrosis. The formation of stress granules is a highly conserved prosurvival response of all eukaryotic cells to type I stress. ${ }^{3}$ Stress granules act as mRNA sorting depots, targeting some mRNA to degradation in P-bodies, stabilizing other mRNAs for translation on stress resolution, and allowing for translation of specific 
A

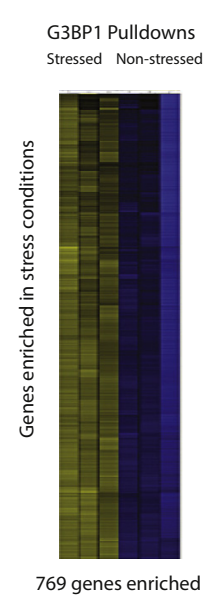

D

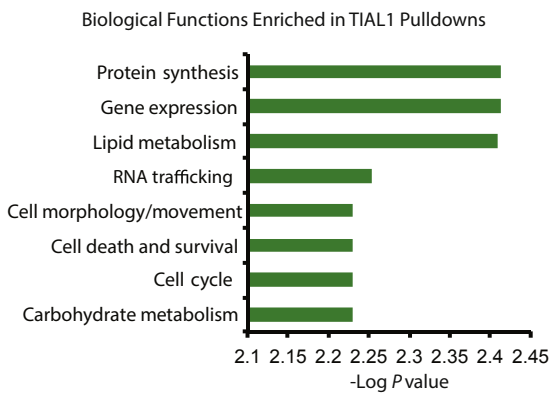

B

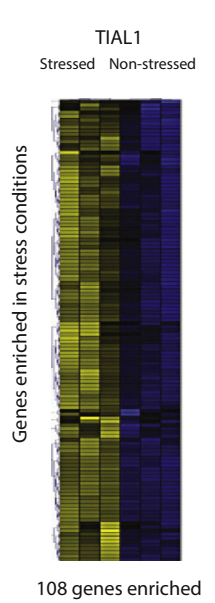

C

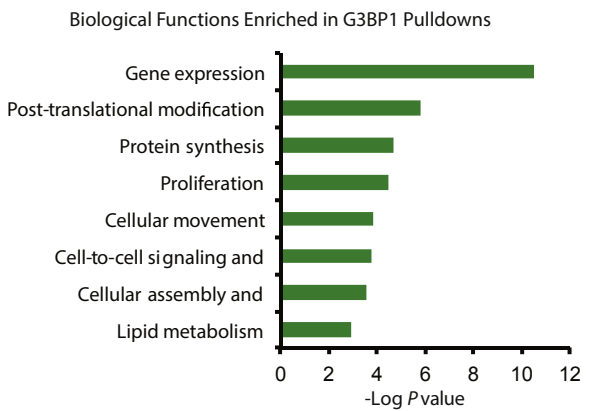

E

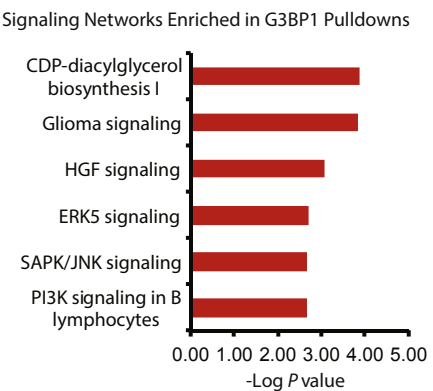

$\mathbf{F}$

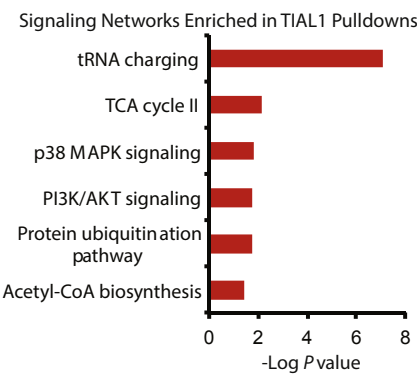

Figure 5 Heat map of the microarray analysis of G3BP1 (A) and TIAR (B) RIPs during arsenite and without arsenite-induced stress. A total of 769 and 108 RNAs were significantly enriched in G3BP1 and TIAR RIPs, respectively. RNAs with a minimum twofold enrichment in stressed versus non-stressed conditions with $P<0.001$ and a false discovery $<5 \%$ were determined as significantly enriched under stress conditions compared with non-stressed conditions using a Bonferroni corrected $t$-test. Yellow represents high signal on the microarray, and blue represents low signal on the microarray. $\mathbf{C}$ and $\mathbf{D}$ : Ingenuity pathway analysis of stress-enriched RNAs in the G3BP1 (C) and TIAR (D) RIPs. A significant pathway was represented with a Z-score of $<2$ or $>2$, which corresponds to $P<0.05$. Enriched RNAs in G3BP1 (E) and TIAR (F) RIPs under stress were interrogated to identify enriched signaling networks with ingenuity pathway analysis. Signaling pathways were considered significant with a Z-score of $<2$ or $>2$, which corresponds to $P<0.05$. AKT, RAC-alpha serine/threonine protein kinase; ERK5, extracellular signal-regulated kinase 5; HGF, hepatocyte growth factor; JNK, c-Jun N-terminal kinase; MAPK, mitogen-activated protein kinase; PI3K, phosphoionsitide 3-Kinase; SAPK, stress activated protein kinase; TCA, tricarboxylic acid cycle.

mRNAs that are required for immediate survival. ${ }^{3,17}$ In addition to the stress granule's role in the mRNA life cycle, stress granules sequester proapoptotic proteins, thereby preventing apoptosis during stress. ${ }^{6,11,19}$ It is conceivable that cancer cells would use stress granules as a means of evading hypoxia, radiation, and chemotherapeutic stress. Indeed, previous studies on mTOR complex and hypoxiainducible factor-1 $\alpha$ support this hypothesis. ${ }^{19,20}$ As a result, targeting stress granules may become an important means of sensitizing cancer cells to treatment.

RhoA and Rac1 have been implicated in stress granule formation and mobility. ${ }^{21,22}$ Our laboratory and others have previously shown that upstream regulators of the RhoGTPases, ECT2 and AurkB, are important to astrocytoma pathogenesis. ${ }^{24-26} \mathrm{We}$, therefore, hypothesized that ECT2 and AurkB also play a role in stress granule formation, and the cellular response to stress.

TIAR is a core component of stress and is used as an immunofluorescence marker of stress granules. ${ }^{43} \mathrm{We}$ demonstrate that ECT2 colocalizes with TIAR during arsenite-induced cellular stress. Endogenous pull-down experiments of ECT2 are enriched for TIAR during arsenite-induced stress. Unfortunately, the reciprocal pull down was unsuccessful, which is not uncommon with endogenous pull downs and may be because of antibody masking or specificity. ECT2 RIPs, but not IgG controls, were able to pull down $\beta$-actin mRNA after stress induction, similar to known stress granule components and RNA binding proteins TIAR and PABP1. Taken together, our data suggest that ECT2 is a component of stress granules.

If ECT2 has a functional role in stress granules, then silencing of ECT2 should lead to an increased sensitivity to chemotherapeutics that can trigger stress granule formation. Baseline apoptosis was measured by cleaved poly (ADP-ribose) polymerase and is higher in cells transfected with ECT2 siRNA over NS siRNA controls. We speculate this is a result of cytokinetic failure or a baseline role 


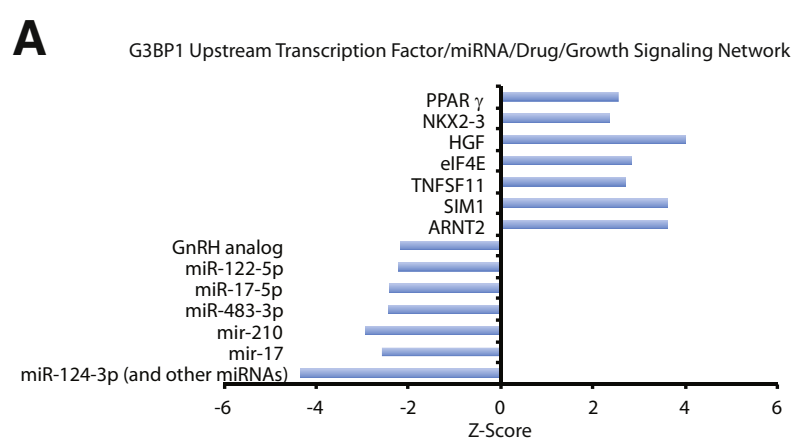

C

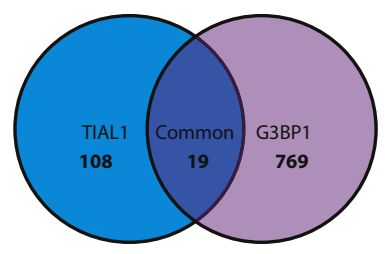

B

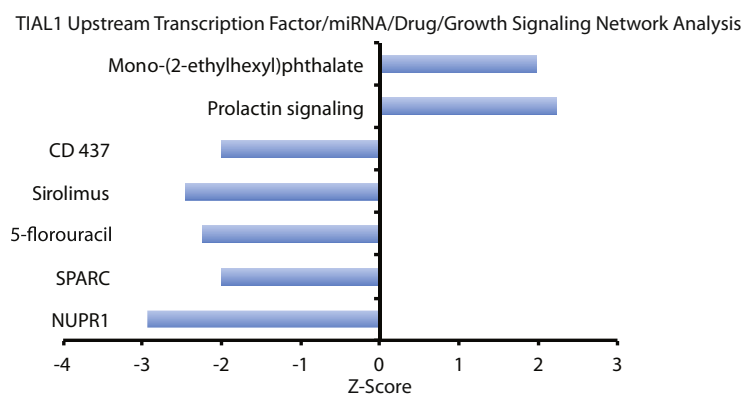

D

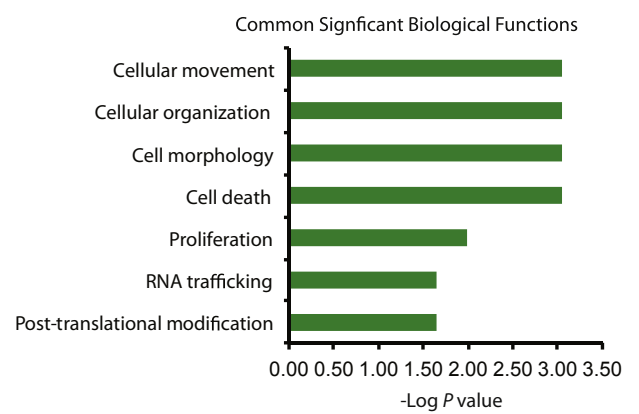

Figure 6 A: Enriched RNAs in G3BP1 pull-down experiments were interrogated to identify enrichment of transcriptional networks, miRNA signaling pathways, growth signaling networks, and pathways altered by drugs. Ingenuity pathway analysis was used to identify significant networks on the basis of classifying RNAs into pathways. Significance of an activated or inhibited pathway was represented with a Z-score of $<2$ or $>2$, which corresponds to $P<0.05$. B: Enriched RNAs in TIAR pull-down experiments were interrogated to identify enrichment of transcriptional networks, miRNA signaling pathways, growth signaling networks, and pathways altered by drugs. Ingenuity pathway analysis was used to identify significant networks on the basis of classifying RNAs into pathways. Significance of an activated or inhibited pathway was represented with a Z-score of $<2$ or $>2$, which corresponds to $P<0.05$. C: Diagram showing the 19 significantly stress-enriched RNAs common to both the TIAR and G3BP1 RIPs. D: Ingenuity pathway analysis of biological functions performed on the 19 commonly enriched RNAs between G3BP1 and TIAR. Significant pathways were represented with a Z-score of $<2$ or $>2$, which corresponds to $P<0.05$. ARNT2, aryl-hydrocarbon receptor nuclear translocator 2; eIF4E, eukaryotic translation initiation factor 4E; HGF, hepatocyte growth factor; NKX2-3, NK2 homeobox 3; PPAR, peroxisome proliferator-activated receptor gamma; SIM1, single-minded homolog 1; TNFSF11, tumour necrosis factor ligand superfamily 11.

of ECT2 in cell survival. To further understand how ECT2 might alter survival, we exposed cells to the chemotherapeutic doxorubicin that induces both stress granules and apoptosis. Cells with loss of ECT2 demonstrated a dramatic sensitization to doxorubicin-induced apoptosis. Although this does not directly link ECT2 to stress granule function, these data demonstrate that ECT2 plays a role in cell survival.

Using a high-throughput cellomics spot assay on the basis of TIAR fluorescence, we showed that siRNA-mediated silencing of ECT2 leads to stress granules in a less mature perinuclear location and a small change in size, but no change in stress granule number or TIAR intensity. Stress granules are highly evolutionarily conserved structures from yeast to mammals, and it is possible that ECT2 loss can be compensated. ${ }^{7}$ There are $>80$ known Rho GTPase pathway guanine nucleotide exchange factors, such as ECT2, with similar structural domains and overlapping functions. It would be interesting to characterize the role of other nucleotide exchange factors within stress granules, and then functionally interrogate multiple guanine nucleotide exchange factors at one time to determine whether the effects are additive.

Given that the cytoskeleton is essential for effective stress granule assembly and cell survival, it is tempting to speculate that ECT2 may play a role in the cytoskeletal rearrangements required to move and sort stress granules similar to the role it plays modifying the cytoskeleton during invasion and cytokinesis. ${ }^{14,44,45}$ The localization of ECT2 to stress granules may modulate granule mobility along the cytoskeleton, given that silencing ECT2 results in fewer granules in a mature perinuclear location. If stress granule movement is impaired, then it is possible that mRNA sorting is dysfunctional, thereby leading to cells with differential sensitivity to stress. Replication of our experiments with loss of multiple guanine nucleotide exchange factors known to play a role in cytoskeleton dynamics may address this hypothesis in future studies.

We hypothesized that a similar regulatory mechanism active during cytokinesis may also be involved in stress granule biology. During cytokinesis, AurkB recruits ECT2 to the cleavage furrow, which, in turn, activates RhoA. ${ }^{45}$ We show, for the first time, that arsenite-induced stress results in phosphorylation of AurkA and AurkB. Pretreatment of cells before stress induction with the aurora kinase inhibitor AZD1152 abolishes AurkA and AurkB phosphorylation and diminishes the amount of p-eIF2 $\alpha$. To confirm that phosphorylated AurkB seen on arseniteinduced stress was functional, we used p-Histone 3 as a surrogate marker for global AurkB activation. Pretreatment 
A

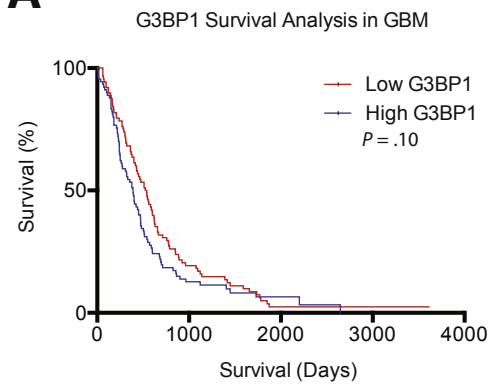

D

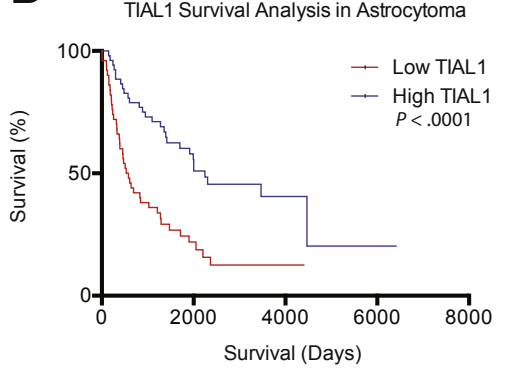

B

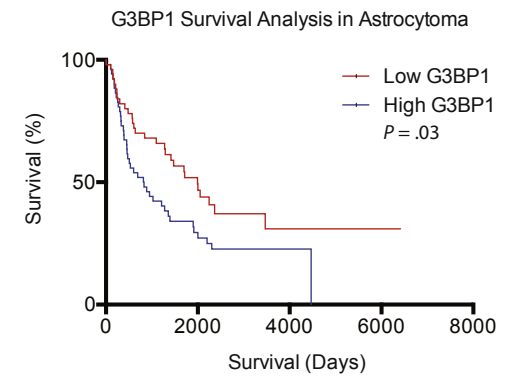

E

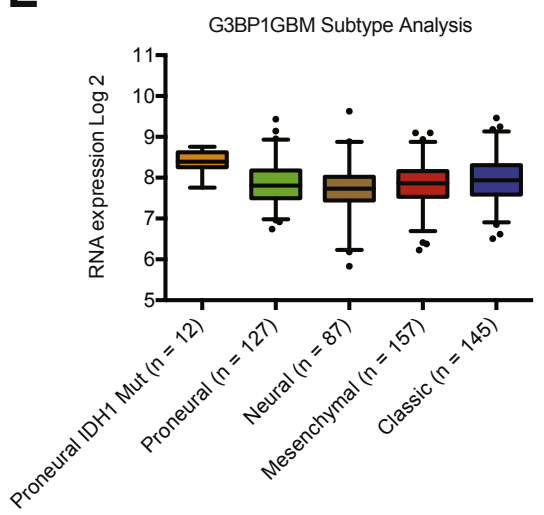

C

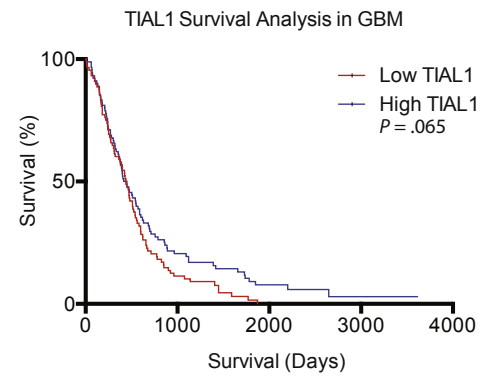

$\mathbf{F}$

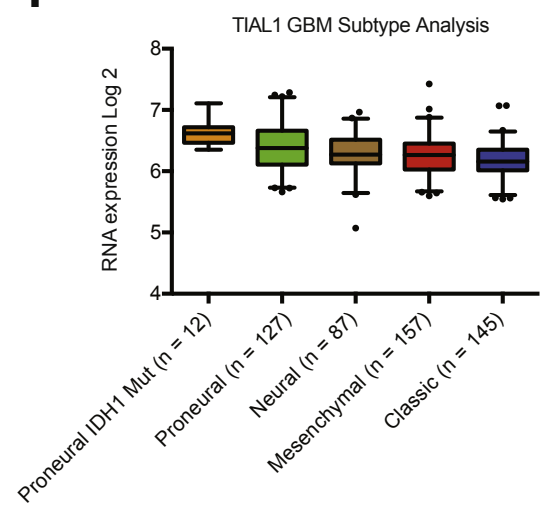

Figure 7 A: Kaplan-Meir survival curve analysis of G3BP1 RNA expression in glioblastoma multiforme (GBM; grade IV) comparing high G3BP1 expression with low G3BP1 expression. B: Kaplan-Meir survival curve analysis of G3BP1 RNA expression in astrocytoma (grades II to III) comparing high G3BP1 expression with low G3BP1 expression. C: Kaplan-Meir survival curve analysis of TIAL1 RNA expression in GBM (grade IV) comparing high TIAL1 expression with low TIAL1 expression. D: Kaplan-Meir survival curve analysis of TIAL1 RNA expression in astrocytoma (grade IV) comparing high TIAL1 expression with low TIAL1 expression. E: G3BP1 gene expression among varying GBM subtypes from the TCGA data set. F: TIAL1 gene expression among varying GBM subtypes from the TCGA data set. $n=89$ (A and C, both groups); $n=51$ (B and $\mathbf{D}$, both groups); $n=528$ (E and $\mathbf{F})$.

of cells before arsenite-induced stress with AZD1152 results in significantly fewer stress granules, smaller stress granules, and less TIAR aggregation, as determined by individual pixel intensity of fluorescence of TIAR in stress granules.

Similar to the aurora kinase inhibitor, AZD1152, AurkBmediated silencing resulted in fewer stress granules, smaller stress granules, and less TIAR aggregation in stress granules. The stress granule effect mediated by AurkB siRNA was not as robust as the chemical inhibitor, and this is likely because of contributions from AurkA, incomplete knockdown, or adaptation to the loss of AurkB over the 3-day knockdown period. We are currently examining the role of AurkA in stress granule biology. AurkA and AurkB share $71 \%$ sequence homology, and we hypothesize that AurkA may also play a role in stress granules. ${ }^{46}$

Only a small portion of cells exhibited colocalization of TIAR and AurkB. Stress granule content is in constant flux during the stress granule life cycle, and despite eIF $2 \alpha$ being key to stress granule initiation, eIF2 $\alpha$ does not colocalize with TIAR in all stress granules (Supplemental Figure S1D).

Taken together, these data suggest that AurkB provides an important signal for stress granule formation. This is intriguing given that inhibition of AurkB renders cancer cells, and specifically astrocytoma cells, more sensitive to chemotherapy and radiotherapy. ${ }^{27,28}$ The demonstration that aurora kinases play a role in stress granule formation provides a potential molecular mechanism underlying this observation. Using drug libraries and cellomics, it may be possible to identify other drugs capable of sensitizing astrocytoma cells to treatment and provide novel therapeutic strategies.

Stress granules are not membrane-bound structures and are, therefore, difficult to isolate using conventional experimental methods. We attempted to enrich for stress granule components using RIPs with TIAR and G3BP1, as described previously. ${ }^{47}$ Stress granules are enriched for the $18 \mathrm{~S}$ ribosomal subunit, but lack the $28 \mathrm{~S}$ subunit. ${ }^{8}$ RNA electropherograms obtained from G3BP1 and TIAR RIPs showed enrichment of the $18 \mathrm{~S}$ subunit with arsenite treatment over arsenite input controls. This demonstrates successful enrichment of the TIAR and G3BP1 components in stress granules. Interestingly, G3BP1 showed enrichment in both arsenite and nonarsenite treatment conditions. This suggests that G3BP1 may only associate with the $48 \mathrm{~S}$ translational initiation complex even during non-stress conditions.

Exon microarray analysis of the RIPs from TIAR and G3BP1 showed enrichment of 108 and 769 RNA species, respectively, over non-stress and input controls in all three biological replicates. Nineteen of these RNAs overlapped. 
Of the 19 overlapping RNAs, 15 have putative AU-rich binding elements in their $3^{\prime}$-UTR, consistent with what is currently known about RNAs targeted to stress granules. ${ }^{48}$ Of the 19 genes, 8 have a known role in stress-related pathways: BEX1 is a signaling protein involved in apoptosis and coordinating external cues into internal signals, CDR1 is a drug transporter implicated in drug resistance, CXADR and THOC7 are involved in the response to viral infection, TAB2 regulates autophagy, MRPL41 and TAB2 are implicated apoptosis pathways, and HIST1H3A and EPC2 are involved in DNA damage and repair. The response to stress is diverse and encompasses many Gene Ontology pathways, such as cellular organization, cell death, RNA trafficking, and gene expression. ${ }^{49-57}$ The lack of significant overlap between RIPs may have been a technical problem. However, more likely this observation represents a differential binding of mRNA to specific stress granule components, because it has been hypothesized that the ultimate fate of mRNA contained within the stress granules may be dictated by protein binding. TIAR has been associated with mRNA degradation. ${ }^{58}$ Thus, one may expect differential enrichment between RIPs of TIAR and G3BP1. Interestingly, analysis of the REMBRANDT Cancer Atlas demonstrates that high expression of G3BP1 levels significantly predicts poor survival, whereas high levels of TIAR expression predict improved survival among astrocytoma patients, although this did not reach statistical significance. This dichotomy would be interesting to explore further with a larger patient population.

The pathway analysis of RNAs isolated from stressinduced RIPs enriches for a diverse array of cellular functions. Pathways involved in cancer biology, such as cell death, cell migration, transcription, and cellular metabolism, were significantly enriched in stress granules. Pathways involved in cellular response to stress, such as lipid and carbohydrate metabolism, cell cycle control, cell death, and survival, were enriched in our RIPs. ${ }^{59}$ Signaling pathway analysis of the genes involved in G3BP1 stress RIPs showed significant enrichment of astrocytoma signaling pathways. The mTOR pathway has been implicated in both astrocytoma pathogenesis and stress granule life cycle. Both the mRNA and protein of mTOR regulators are contained in stress granules. ${ }^{19}$ G3BP1 RIPs showed an enrichment of mRNAs targeted by sirolimus, a known chemotherapeutic and inhibitor of mTOR. Known genes involved in GBM signaling biology were identified in G3BP1 stress immunoprecipitations, such as PTEN, TGFb, phosphatidylinositol 3-kinases, mitogen-activated protein kinase, and MDM2. Interestingly, proteins associated with RhoGTPases were enriched in G3BP1 stress granules, such as TIAM1, WAVE1-3, LARG (ARGHGEF12), ARHGAP11, 12, 29, and 32, and LIMK.

This is the first study to examine the contents of stress granules in astrocytomas. The expression level of these proteins before, during, and after stress needs to be studied further to aid in determining stress granule contribution to pathway member expression and pathogenesis. For example, hypoxia-inducible factor $1 \alpha$ transcripts are stabilized in stress granules during radiation for rapid translation during the reoxygenation phase in cancer cells. ${ }^{20}$ It is possible that RhoGTPase transcripts are also stabilized in stress granules until acclimatization to stress, after which there may be immediate translation to allow for cellular migration away from a stressful microenvironment. This is an active area of research in our laboratory.

Interestingly, G3BP1 stress RIPs also demonstrate an enrichment of genes that are a target of miRNA degradation. Most of these miRNAs are involved in cancer, such as the miR-17, miR-122, miR-124, miR-210, miR-483, and more specifically, miR-17 and miR-124 in GBM. ${ }^{60-64}$ The relationship between miRNAs and stress granule-enriched genes is not surprising. Stress granules have a functional link with the foci of mRNA degradation, P-bodies, during stress. Whether these genes are stabilized for translation on stress adaptation or will be eventually targeted to P-bodies remains to be elucidated. However, stress granules provide a haven for specific mRNAs during stress. The enrichment of specific miRNA target genes demonstrates this may be an alternate pathway for miRNA pathway dysfunction in astrocytoma cells.

Although not an exhaustive list of stress granule contents, our data confirm that RIP technology can be used to identify stress granule mRNA components in astrocytoma cells. Notably, this technique is limited to antibody specificity because G3BP1 pulled down more mRNA species than TIAR. Our enrichment criteria were strict; thus, if an mRNA bound to TIAR and G3BP1 during non-stressed and stressed conditions, it would have been eliminated from the analysis despite being contained within stress granules and enriched over input controls. This criterion was specifically designed to eliminate non-specific effects of the antibody. It would be interesting to repeat these experiments with other stress granule components, such as HuR, given that HuR is overexpressed in astrocytoma and is a known mRNA stabilizer. ${ }^{65}$ In the future, we would like to compare normal human brain specimens with astrocytoma tumor specimens to determine whether there is a difference in mRNA sorting that can be pursued as a therapeutic target.

In summary, we have shown a novel role for ECT2 and the aurora kinases in stress granule biology. Our data further identify these proteins as potential targets for astrocytoma therapy. The role of stress granules in astrocytoma and cancer biology is an emerging field of investigative inquiry, and warrants further study as we begin to understand the epigenetic modifications that allow cancer cells to adapt to and evade stress.

\section{Acknowledgment}

We thank Dr. Peter Dirks (The Hospital for Sick Children, Toronto, ON, Canada) for providing the glioblastoma multiforme adherent progenitor cells. 


\section{Supplemental Data}

Supplemental material for this article can be found at http://dx.doi.org/10.1016/j.ajpath.2016.02.013.

\section{References}

1. Yamasaki S, Anderson P: Reprogramming mRNA translation during stress. Curr Opin Cell Biol 2008, 20:222-226

2. Holcik M, Sonenberg N: Translational control in stress and apoptosis. Nat Rev Mol Cell Biol 2005, 6:318-327

3. Thomas MG, Loschi M, Desbats MA, Boccaccio GL: RNA granules: the good, the bad and the ugly. Cell Signal 2011, 23:324-334

4. Kedersha N, Anderson P: Stress granules: sites of mRNA triage that regulate mRNA stability and translatability. Biochem Soc Trans 2002, 30:963-969

5. Gilks N, Kedersha N, Ayodele M, Shen L, Stoecklin G, Dember LM, Anderson P: Stress granule assembly is mediated by prion-like aggregation of TIA-1. Mol Biol Cell 2004, 15:5383-5398

6. Kedersha N, Anderson P: Regulation of translation by stress granules and processing bodies. Prog Mol Biol Transl Sci 2009, 90:155-185

7. Anderson P, Kedersha N: Stress granules: the Tao of RNA triage. Trends Biochem Sci 2008, 33:141-150

8. Souquere S, Mollet S, Kress M, Dautry F, Pierron G, Weil D: Unravelling the ultrastructure of stress granules and associated P-bodies in human cells. J Cell Sci 2009, 122:3619-3626

9. Matsuki H, Takahashi M, Higuchi M, Makokha GN, Oie M, Fujii M: Both G3BP1 and G3BP2 contribute to stress granule formation. Genes Cells 2013, 18:135-146

10. Ivanov P, Kedersha N, Anderson P: Stress puts TIA on TOP. Genes Dev 2011, 25:2119-2124

11. Anderson P, Kedersha N: Stress granules. Curr Biol 2009, 19: R397-R398

12. Kolobova E, Efimov A, Kaverina I, Rishi AK, Schrader JW, Ham AJ, Larocca MC, Goldenring JR: Microtubule-dependent association of AKAP350A and CCAR1 with RNA stress granules. Exp Cell Res 2009, 315:542-555

13. Fujimura K, Katahira J, Kano F, Yoneda Y, Murata M: Microscopic dissection of the process of stress granule assembly. Biochim Biophys Acta 2009, 1793:1728-1737

14. Ivanov PA, Chudinova EM, Nadezhdina ES: Disruption of microtubules inhibits cytoplasmic ribonucleoprotein stress granule formation. Exp Cell Res 2003, 290:227-233

15. Loschi M, Leishman CC, Berardone N, Boccaccio GL: Dynein and kinesin regulate stress-granule and P-body dynamics. J Cell Sci 2009 122:3973-3982

16. von Roretz C, Di Marco S, Mazroui R, Gallouzi IE: Turnover of AUrich-containing mRNAs during stress: a matter of survival. Wiley Interdiscip Rev RNA 2011, 2:336-347

17. Ohn T, Anderson P: The role of posttranslational modifications in the assembly of stress granules. Wiley Interdiscip Rev RNA 2010, 1 486-493

18. Anderson P, Kedersha N, Ivanov P: Stress granules, P-bodies and cancer. Biochim Biophys Acta 2015, 1849:861-870

19. Thedieck K, Holzwarth B, Prentzell MT, Boehlke C, Klasener K, Ruf S, Sonntag AG, Maerz L, Grellscheid SN, Kremmer E, Nitschke R, Kuehn EW, Jonker JW, Groen AK, Reth M, Hall MN, Baumeister R: Inhibition of mTORC1 by astrin and stress granules prevents apoptosis in cancer cells. Cell 2013, 154:859-874

20. Moeller BJ, Cao Y, Li CY, Dewhirst MW: Radiation activates HIF-1 to regulate vascular radiosensitivity in tumors: role of reoxygenation, free radicals, and stress granules. Cancer Cell 2004, 5:429-441

21. Tsai NP, Wei LN: RhoA/ROCK1 signaling regulates stress granule formation and apoptosis. Cell Signal 2010, 22:668-675
22. Sahoo PK, Murawala P, Sawale PT, Sahoo MR, Tripathi MM, Gaikwad SR, Seshadri V, Joseph J: Wnt signalling antagonizes stress granule assembly through a Dishevelled-dependent mechanism. Biol Open 2012, 1:109-119

23. Petronczki M, Glotzer M, Kraut N, Peters JM: Polo-like kinase 1 triggers the initiation of cytokinesis in human cells by promoting recruitment of the RhoGEF Ect2 to the central spindle. Dev Cell 2007, 12:713-725

24. Weeks A, Okolowsky N, Golbourn B, Ivanchuk S, Smith C, Rutka JT: ECT2 and RASAL2 mediate mesenchymal-amoeboid transition in human astrocytoma cells. Am J Pathol 2012, 181:662-674

25. Salhia B, Tran NL, Chan A, Wolf A, Nakada M, Rutka F, Ennis M, McDonough WS, Berens ME, Symons M, Rutka JT: The guanine nucleotide exchange factors trio, Ect2, and Vav3 mediate the invasive behavior of glioblastoma. Am J Pathol 2008, 173:1828-1838

26. Diaz RJ, Golbourn B, Shekarforoush M, Smith CA, Rutka JT: Aurora kinase $\mathrm{B} / \mathrm{C}$ inhibition impairs malignant glioma growth in vivo. Neurooncol 2012, 108:349-360

27. Borges KS, Castro-Gamero AM, Moreno DA, da Silva Silveira V, Brassesco MS, de Paula Queiroz RG, de Oliveira HF, Carlotti CG Jr, Scrideli CA, Tone LG: Inhibition of Aurora kinases enhances chemosensitivity to temozolomide and causes radiosensitization in glioblastoma cells. J Cancer Res Clin Oncol 2012, 138:405-414

28. Azzariti A, Bocci G, Porcelli L, Fioravanti A, Sini P, Simone GM, Quatrale AE, Chiarappa P, Mangia A, Sebastian S, Del Bufalo D, De Tacca M, Paradiso A: Aurora B kinase inhibitor AZD1152: determinants of action and ability to enhance chemotherapeutics effectiveness in pancreatic and colon cancer. Br J Cancer 2011, 104:769-780

29. Hanahan D, Weinberg RA: Hallmarks of cancer: the next generation. Cell 2011, 144:646-674

30. Pollard SM, Yoshikawa K, Clarke ID, Danovi D, Stricker S, Russell R, Bayani J, Head R, Lee M, Bernstein M, Squire JA, Smith A, Dirks P: Glioma stem cell lines expanded in adherent culture have tumor-specific phenotypes and are suitable for chemical and genetic screens. Cell Stem Cell 2009, 4:568-580

31. Walsby E, Walsh V, Pepper C, Burnett A, Mills K: Effects of the aurora kinase inhibitors AZD1152-HQPA and ZM447439 on growth arrest and polyploidy in acute myeloid leukemia cell lines and primary blasts. Haematologica 2008, 93:662-669

32. Smith CA, Dho SE, Donaldson J, Tepass U, McGlade CJ: The cell fate determinant numb interacts with EHD/Rme-1 family proteins and has a role in endocytic recycling. Mol Biol Cell 2004, 15:3698-3708

33. Gruber AR, Fallmann J, Kratochvill F, Kovarik P, Hofacker IL: AREsite: a database for the comprehensive investigation of AU-rich elements. Nucleic Acids Res 2011, 39:D66-D69

34. Brennan CW, Verhaak RG, McKenna A, Campos B, Noushmehr H, Salama SR, et al: The somatic genomic landscape of glioblastoma Cell 2013, 155:462-477

35. Madhavan S, Zenklusen JC, Kotliarov Y, Sahni H, Fine HA Buetow K: Rembrandt: helping personalized medicine become a reality through integrative translational research. Mol Cancer Res 2009, 7:157-167

36. Verhaak RG, Hoadley KA, Purdom E, Wang V, Qi Y, Wilkerson MD, Miller CR, Ding L, Golub T, Mesirov JP, Alexe G, Lawrence M, O'Kelly M, Tamayo P, Weir BA, Gabriel S, Winckler W, Gupta S, Jakkula L, Feiler HS, Hodgson JG, James CD, Sarkaria JN, Brennan C, Kahn A, Spellman PT, Wilson RK, Speed TP, Gray JW, Meyerson M, Getz G, Perou CM, Hayes DN Cancer Genome Atlas Research Network: Integrated genomic analysis identifies clinically relevant subtypes of glioblastoma characterized by abnormalities in PDGFRA, IDH1, EGFR, and NF1. Cancer Cell 2010, 17:98-110

37. Kedersha NL, Gupta M, Li W, Miller I, Anderson P: RNA-binding proteins TIA-1 and TIAR link the phosphorylation of eIF-2 alpha to the assembly of mammalian stress granules. J Cell Biol 1999, 147: $1431-1442$ 
38. Costes SV, Daelemans D, Cho EH, Dobbin Z, Pavlakis G, Lockett S: Automatic and quantitative measurement of protein-protein colocalization in live cells. Biophys J 2004, 86:3993-4003

39. Nishimura Y, Yonemura S: Centralspindlin regulates ECT2 and RhoA accumulation at the equatorial cortex during cytokinesis. J Cell Sci 2006, 119:104-114

40. Kimura K, Tsuji T, Takada Y, Miki T, Narumiya S: Accumulation of GTP-bound RhoA during cytokinesis and a critical role of ECT2 in this accumulation. J Biol Chem 2000, 275:17233-17236

41. Chalamalasetty RB, Hummer S, Nigg EA, Sillje HH: Influence of human Ect2 depletion and overexpression on cleavage furrow formation and abscission. J Cell Sci 2006, 119:3008-3019

42. Morita T, Satoh R, Umeda N, Kita A, Sugiura R: The stress granule protein Vg11 and poly(A)-binding protein Pab1 are required for doxorubicin resistance in the fission yeast Schizosaccharomyces pombe. Biochem Biophys Res Commun 2012, 417:399-403

43. Anderson P, Kedersha N: Visibly stressed: the role of eIF2, TIA-1, and stress granules in protein translation. Cell Stress Chaperones 2002, 7:213-221

44. Nadezhdina ES, Lomakin AJ, Shpilman AA, Chudinova EM, Ivanov PA: Microtubules govern stress granule mobility and dynamics. Biochim Biophys Acta 2010, 1803:361-371

45. Yuce O, Piekny A, Glotzer M: An ECT2-centralspindlin complex regulates the localization and function of RhoA. J Cell Biol 2005, 170:571-582

46. Bolanos-Garcia VM: Aurora kinases. Int J Biochem Cell Biol 2005, $37: 1572-1577$

47. Mazan-Mamczarz K, Lal A, Martindale JL, Kawai T, Gorospe M: Translational repression by RNA-binding protein TIAR. Mol Cell Biol 2006, 26:2716-2727

48. Buchan JR, Parker R: Eukaryotic stress granules: the ins and outs of translation. Mol Cell 2009, 36:932-941

49. Xiao Q, Hu Y, Liu Y, Wang Z, Geng H, Hu L, Xu D, Wang K, Zheng L, Zheng S, Ding K: BEX1 promotes imatinib-induced apoptosis by binding to and antagonizing BCL-2. PLoS One 2014, 9:e91782

50. Prasad R, Banerjee A, Khandelwal NK, Dhamgaye S: The ABCs of Candida albicans multidrug transporter Cdr1. Eukaryot Cell 2015, 14 : 1154-1164

51. Bergelson JM, Cunningham JA, Droguett G, Kurt-Jones EA, Krithivas A, Hong JS, Horwitz MS, Crowell RL, Finberg RW: Isolation of a common receptor for Coxsackie B viruses and adenoviruses 2 and 5. Science 1997, 275:1320-1323

52. Sakuma T, Tonne JM, Ikeda Y: Murine leukemia virus uses TREX components for efficient nuclear export of unspliced viral transcripts. Viruses 2014, 6:1135-1148

53. Song L, Gu Y, Jie J, Bai X, Yang Y, Liu C, Liu Q: Dab2 attenuates brain injury in APP/PS1 mice via targeting transforming growth factor-beta/SMAD signaling. Neural Regen Res 2014, 9:41-50
54. Criollo A, Niso-Santano M, Malik SA, Michaud M, Morselli E, Marino G, Lachkar S, Arkhipenko AV, Harper F, Pierron G, Rain JC, Ninomiya-Tsuji J, Fuentes JM, Lavandero S, Galluzzi L, Maiuri MC, Kroemer G: Inhibition of autophagy by TAB2 and TAB3. EMBO J 2011, 30:4908-4920

55. Sanjo H, Takeda K, Tsujimura T, Ninomiya-Tsuji J, Matsumoto K, Akira S: TAB2 is essential for prevention of apoptosis in fetal liver but not for interleukin-1 signaling. Mol Cell Biol 2003, 23: $1231-1238$

56. Gong F, Miller KM: Mammalian DNA repair: HATs and HDACs make their mark through histone acetylation. Mutat Res 2013, 750: 23-30

57. Malladi S, Parsa KV, Bhupathi D, Rodriguez-Gonzalez MA, Conde JA, Anumula P, Romo HE, Claunch CJ, Ballestero RP, Gonzalez-Garcia M: Deletion mutational analysis of BMRP, a proapoptotic protein that binds to Bcl-2. Mol Cell Biochem 2011, 351: 217-232

58. Podszywalow-Bartnicka P, Wolczyk M, Kusio-Kobialka M, Wolanin K, Skowronek K, Nieborowska-Skorska M, Dasgupta Y, Skorski T, Piwocka K: Downregulation of BRCA1 protein in BCRABL1 leukemia cells depends on stress-triggered TIAR-mediated suppression of translation. Cell Cycle 2014, 13:3727-3741

59. Kultz D: Molecular and evolutionary basis of the cellular stress response. Annu Rev Physiol 2005, 67:225-257

60. Yoshino H, Seki N, Itesako T, Chiyomaru T, Nakagawa M, Enokida H: Aberrant expression of microRNAs in bladder cancer. Nat Rev Urol 2013, 10:396-404

61. Kandalam MM, Beta M, Maheswari UK, Swaminathan S, Krishnakumar S: Oncogenic microRNA 17-92 cluster is regulated by epithelial cell adhesion molecule and could be a potential therapeutic target in retinoblastoma. Mol Vis 2012, 18:2279-2287

62. Bertero T, Bourget-Ponzio I, Puissant A, Loubat A, Mari B, Meneguzzi G, Auberger P, Barbry P, Ponzio G, Rezzonico R: Tumor suppressor function of miR-483-3p on squamous cell carcinomas due to its pro-apoptotic properties. Cell Cycle 2013, 12: $2183-2193$

63. Ohno M, Ohkuri T, Kosaka A, Tanahashi K, June CH, Natsume A, Okada H: Expression of miR-17-92 enhances anti-tumor activity of T-cells transduced with the anti-EGFRvIII chimeric antigen receptor in mice bearing human GBM xenografts. J Immunother Cancer 2013, $1: 21$

64. Karsy M, Arslan E, Moy F: Current progress on understanding microRNAs in glioblastoma multiforme. Genes Cancer 2012, 3: $3-15$

65. Nabors LB, Gillespie GY, Harkins L, King PH: HuR, a RNA stability factor, is expressed in malignant brain tumors and binds to adenineand uridine-rich elements within the 3' untranslated regions of cytokine and angiogenic factor mRNAs. Cancer Res 2001, 61: $2154-2161$ 